\title{
Modeling the dust Spectral Energy Distribution of NGC 4214
}

\author{
Israel Hermelo ${ }^{1}$, Ute Lisenfeld ${ }^{1}$, Mónica Relaño ${ }^{1}$, Richard J. Tuffs ${ }^{2}$, \\ Cristina C. Popescu ${ }^{3}$, Jörg Fischera ${ }^{4}$ and Brent Groves ${ }^{5}$ \\ ${ }^{1}$ Departamento de Física Teórica y del Cosmos, Universidad de Granada, Spain \\ email: israelhermelo@ugr.es, ute@ugr.es, mrelano@ugr.es \\ ${ }^{2}$ Max Planck Institut für Kernphysik, Heidelberg, Germany \\ email: Richard.Tuffs@mpi-hd.mpg.de \\ ${ }^{3}$ Jeremiah Horrocks Institute for Astrophysics and Supercomputing, University of Central \\ Lancashire, Preston, U.K. \\ email: cpopescu@uclan.ac.uk \\ ${ }^{4}$ Canadian Institute for Theoretical Astrophysics (CITA), University of Toronto \\ email: fischera@cita.utoronto.ca \\ ${ }^{5}$ Max Planck Institut für Astronomie, Heidelberg, Germany \\ email: brent@mpia.de
}

\begin{abstract}
We have carried out a detailed modeling of the dust Spectral Energy Distribution (SED) of the nearby, starbursting dwarf galaxy NGC 4214. A key point of our modeling is that we distinguish the emission from (i) HII regions and their associated photodissociation regions (PDRs) and (ii) diffuse dust. For both components we apply templates from the literature calculated with a realistic geometry and including radiation transfer. The large amount of existing data from the ultraviolet (UV) to the radio allows the direct measurement of most of the input parameters of the models. We achieve a good fit for the total dust SED of NGC 4214. In the present contribution we describe the available data, the data reduction and the determination of the model parameters, whereas a description of the general outline of our work is presented in the contribution of Lisenfeld et al. in this volume.
\end{abstract}

Keywords. dust, extinction, galaxies: irregular, galaxies: individual (NGC 4214), galaxies: ISM

\section{Introduction}

NGC 4214 is a nearby (2.94 Mpc; Maíz-Apellániz et al. 2002) barred Magellanic irregular galaxy consisting of a smooth extended disk (Figure 1a) and a centrally concentrated young star forming (SF) region dominated by the two SF complexes NW and SE (Figure 1b). The metallicity of both complexes has been measured by Kobulnicky \& Skillman (1996), who found $Z \sim 0.3 Z_{\odot}$. The main stellar cluster in the NW complex, referred here as NW-A, is located at the center of the galaxy and has removed most of the gas in our line of sight. Using stellar synthesis models, Úbeda et al. (2007) determined the age, the mass, the radius and the extinction of the stellar clusters within the complexes NW and SE.

We have modeled the dust SED of NGC 4214 separately for the dust emission from SF regions and from the diffuse component. Due to its proximity, the large amount and good quality of the available data and previous studies of this object, most of the model parameters can be directly calculated, making this galaxy an excellent laboratory to improve our knowledge about dust in dwarf galaxies. 


\section{Photometry}

In order to determine the observational dust SED of NGC 4214 we used data from Spitzer, Herschel, and Planck archives, as well as our own observations at $1200 \mu \mathrm{m}$ using the MAMBO bolometer at the IRAM 30m telescope on Pico Veleta (Spain). For all the photometric measurements (i) color corrections, (ii) aperture corrections, and (iii) line decontamination were done. With the exception of the atomic line [CII] $158 \mu \mathrm{m}$, which contributes $4.26 \%$ to the flux of MIPS $160 \mu \mathrm{m}$, the contamination of the other considered lines ([OI] 63, [OIII] 88, [NII] 122, [OI] 146 and [NII] $205 \mu \mathrm{m}$ as well as CO rotational lines) was found to be negligible. Besides, the stellar contribution in the IRAC $8 \mu \mathrm{m}$ image was subtracted. Figures $1 \mathrm{a}$ and $1 \mathrm{~b}$ show the apertures used to measure the total emission and the emission from the SF complexes, respectively. In order to constrain the thermal radio emission we made use of the extinction corrected $\mathrm{H} \alpha$ flux reported by MacKenty et al. (2000) and eq. (3) and (4a) in Condon (1992). The diffuse dust SED was determined by subtracting the combined emission of NW and SE complexes from the total emission of the galaxy.

\section{Determination of the parameters}

To model the dust emission from the HII regions we used the model from Groves et al. (2008) based on radiation transfer calculations. This model computes the whole emergent spectrum including the attenuated stellar spectrum, the line emission spectrum, as well as a dust SED. The input parameters of this model are (i) the age of the stellar cluster, (ii) the metallicity, Z, (iii) the hydrogen column density of the surrounding PDR, $N_{\mathrm{H}}$, (iv) the external pressure, $p_{0}$, (v) the compactness, $C$, which parametrizes the dust grain temperature distribution, and (vi) the covering factor, $f_{\text {cov }}$, which represents the fraction of the surface of the HII region which is covered by the PDR. With exception of $N_{\mathrm{H}}$, all the other parameters were observationally determined:

- Following Úbeda et al. (2007), we adopted 5 and 3.5 Myr for the age of the NW and SE complex, respectively.

- We used a metallicity of $\mathrm{Z}=0.2 \mathrm{Z}_{\odot}$ (results shown here) and also tested $\mathrm{Z}=0.4 \mathrm{Z}_{\odot}$ which yielded very similar results (no template for $\mathrm{Z}=0.3 \mathrm{Z}_{\odot}$ is available).

- We determined $p_{0}$ by comparing the expected and the observed radii of the HII regions as a function of the age. We found values of $\log \left[\left(p_{0} / k\right) / \mathrm{cm}^{-3} \mathrm{~K}\right] \sim 6-7$.

- $C$ was directly determined from the external pressure and the mass of the clusters. We found values of 5.0-6.0 and 4.5-5.5 for the NW and SE complexes, respectively.

- In order to calculate $f_{\text {cov }}$ we assumed an optically thick, uniformly fragmented PDR surrounding the ionized gas. With these assumptions, the intrinsic luminosity of the central stellar cluster can be approximated by the sum of the observed luminosities of the stars, $L_{s}$, and the luminosity re-emitted by the dust, $L_{d}$. In this picture, the covering factor is $f_{\text {cov }}=\frac{L_{d}}{L_{d}+L_{s}}$. We obtained $f_{\text {cov }}=0.44$ and 0.65 for NW and SE, respectively.

To model the diffuse dust emission we used the model from Popescu et al. (2011) based on full radiation transfer calculations of the propagation of starlight in galaxy disks. The relevant parameters of this model in the case of NGC 4214 are (i) the radial B-band scale-length of the old stellar disk, $h_{s}$, (ii) the central B-band face-on opacity, $\tau_{B}^{f}$, (iii) the star formation rate, $S F R$, (iv) the luminosity of the old stellar population, old, and (v) the clumpiness factor, $F$, which is equivalent to the covering factor of the Groves et al. (2008) model. From the primary parameters $S F R$ and F, Popescu et al. (2011) define the star formation rate powering the diffuse dust emission as $S F R^{\prime}=S F R \times(1-F)$. The diffuse SED, as well as the parameters $S F R^{\prime}$ and old, which control the radiation 
field, must be scaled by comparing the scale-length of NGC 4214 with the reference scale-length $h_{s}^{\text {ref }}=5670 \mathrm{pc}$. In our case, we observationally determined:

- The scale-length, for which we obtained $h_{s}=873_{-123}^{+172} \mathrm{pc}$ by fitting elliptical isophotes.

- The clumpiness factor, which was calculated as the average covering factor of the NW and SE regions $\left(<f_{\text {cov }}>=0.55\right)$.

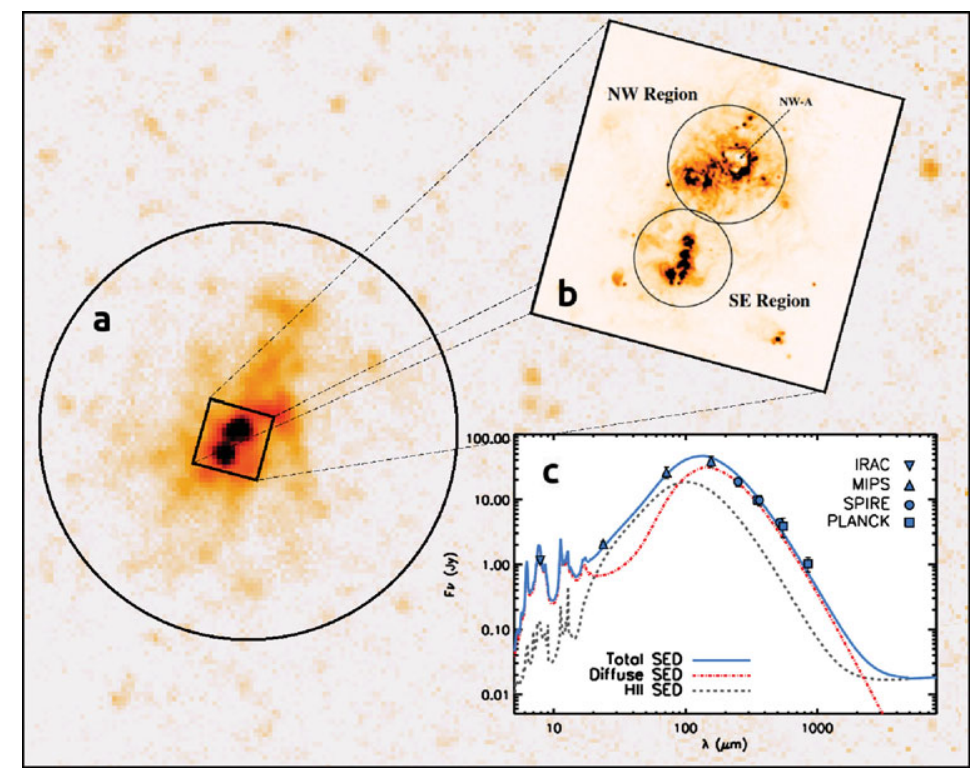

Figure 1. a) SPIRE $250 \mu \mathrm{m}$ image of the disk of NGC 4214. The circle corresponds to the aperture we used to measure the global emission and it has a radius of $\sim 4 \mathrm{kpc}$. b) HST-WFC3 $\mathrm{H} \alpha$ map showing the complexes NW and SE and the location of the SSC NW-A. c) Best-fit model (solid line) to the global SED for the parameters described in section 3. The global SED was obtained as the sum of the best-fits for the complexes NW and SE (dashed line) and the best-fit for the diffuse dust (dashed-dotted line).

\section{Results}

Figure 1c shows the data points of the total observed dust SED, together with the bestfit model (solid line) calculated as the sum of the models of the SF regions (dashed line) and the model of the diffuse dust (dashed-dotted line). The fits to the emission of the SF regions were obtained individually using the parameters described in Sec. 3, whereas the fit to the diffuse emission was obtained leaving $\tau_{B}^{f}, S F R^{\prime}$, and old as free parameters. We achieved a good agreement between the observed and the modelled FIR/submm SED of NGC 4214. However, from our SED modelling we inferred $S F R=0.16 M_{\odot} \mathrm{yr}^{-1}$ which is a factor 1.7 smaller than the value derived from the observed, integrated UV-to-optical luminosity, indicating that part of the stellar radiation does not contribute to the diffuse dust heating (see Lisenfeld et al. in this volume for more details).

\section{References}

Condon, J. J. 1992, ARAA, 30, 575

Groves, B., Dopita, M. A., Sutherland, R. S., et al. 2008, ApJS, 176, 438

Kobulnicky, H. A. \& Skillman, E. D. 1996, ApJ, 471, 211

Maíz-Apellániz, J., Cieza, L., \& MacKenty, J. W. 2002, AJ, 123, 1307

MacKenty, J. W., Maíz-Apellániz, J., Pickens, C. E., et al. 2000, AJ, 120, 3007

Popescu, C. C., Tuffs, R. J., Dopita, M. A. et al. 2011, A\&A, 527, A109

Úbeda, L., Maíz-Apellániz, J., \& MacKenty, J. W. 2007, AJ, 133, 932 